\title{
Ionization Efficiency Study for Low Energy Nuclear Recoils in Germanium
}

\author{
D. Barker ${ }^{\mathrm{a}}$, W.-Z. Wei ${ }^{\mathrm{a}}$, D.-M. Mei ${ }^{\mathrm{a}, *}$, and C. Zhang ${ }^{\mathrm{a}, \mathrm{b}}$ \\ ${ }^{a}$ Department of Physics, The University of South Dakota, Vermillion, South \\ Dakota 57069 \\ ${ }^{\mathrm{b}}$ College of Sciences, China Three Gorges University, Yichang 443002, China
}

\begin{abstract}
We used the internal conversion ( $E_{0}$ transition) of germanium-72 to indirectly measure the low energy nuclear recoils of germanium. Together with a reliable Monte Carlo package, in which we implement the internal conversion process, the data was compared to the Lindhard $(k=0.159)$ and Barker-Mei models. A shape analysis indicates that both models agree well with data in the region of interest within $4 \%$. The most probable value (MPV) of the nuclear recoils obtained from the shape analysis is $17.5 \pm 0.12$ (sys) \pm 0.035 (stat) $\mathrm{keV}$ with an average path-length of 0.014 $\mu \mathrm{m}$.
\end{abstract}

Key words: Nuclear Recoil, Ionization Efficiency, Dark Matter Detection PACS: 95.35.+d, 07.05.Tp, 25.40.Fq, 29.40.Wk

\section{Introduction}

Understanding detector response to low energy nuclear recoils is imperative to the interpretation of experimental results from a detector designed to search for WIMPs (weakly interacting massive particles), a dark matter candidate. Direct detection of low mass WIMPs occurs in the low energy region of detectors with a threshold down to sub keV. Since the threshold energy represents the visible energy in the detector, understanding the ionization efficiency of the detector response to low energy nuclear recoils is crucial to calculating the recoil energy. An example of the need for this requirement is the claim of experimental evidence for dark matter by CoGeNT [1] that has been unverified by

\footnotetext{
* Corresponding author.

Email address: Dongming. Mei@usd.edu (D.-M. Mei).
} 
CDMS II [2]. Both experiments use germanium as the target material. Thus, similar results are expected if the detection thresholds for both experiments were determined using a standardized ionization efficiency, which accurately accounts for all processes that occur at a low energy range. This standardized ionization efficiency must also be validated with measurements in combination with reliable Monte Carlo simulations.

Two different approaches can be used for modeling ionization efficiency in germanium detectors [3,4]. One model, traditionally used for a number of different detector materials, is proposed by Lindhard et. al. [3]:

$$
\varepsilon=\frac{k \cdot g(\epsilon)}{1+k \cdot g(\epsilon)}
$$

where $k=0.133 Z^{2 / 3} A^{-1 / 2}, g(\epsilon)=3 \epsilon^{0.15}+0.7 \epsilon^{0.6}+\epsilon$, and $\epsilon=11.5 E_{r} Z^{-7 / 3}$ for a given atomic number, $Z$, mass number, $A$, and recoil energy, $E_{r}$. However, this model has not been proved accurate at low energies as the theoretical derivation has uncertainties in this region [3].

Another model designed for low energy interactions in germanium was proposed by Barker and Mei [4]. This model takes into account the fraction of nuclear stopping power that contributes to the ionization efficiency at low energies [4]. The Barker-Mei model can be expressed as:

$$
\varepsilon_{c}=\frac{0.14476 \cdot E_{r}^{0.697747}}{-1.8728+\exp \left[E_{r}^{0.211349}\right]} .
$$

This model is valid for recoil energies, $E_{r}$, from $1 \mathrm{keV}$ to $100 \mathrm{keV}$. However, the Barker-Mei model has not been experimentally verified. The purpose of this paper is to address that issue.

A comparison between the two models and existing data was performed [4], and Fig. 1 shows both models together with available experimental data. As shown in Fig. 1, the two models agree in the low energy region but disagree with the available data points in the same region. Further validation of the Lindhard and Barker-Mei models, using more measurements and more accurate Monte Carlo simulation, was necessary.

Taking exact nuclear recoil measurements can be challenging at lower energies when systematic errors are introduced by a variety of sources. For example, thermal neutrons and elastic/inelastic scattering have their own uncertainties. Utilizing thermal neutrons requires the full absorption of out-going gamma rays measured by another detector in coincidence. Without this additional measurement, Compton scattering from out-going gamma rays within 


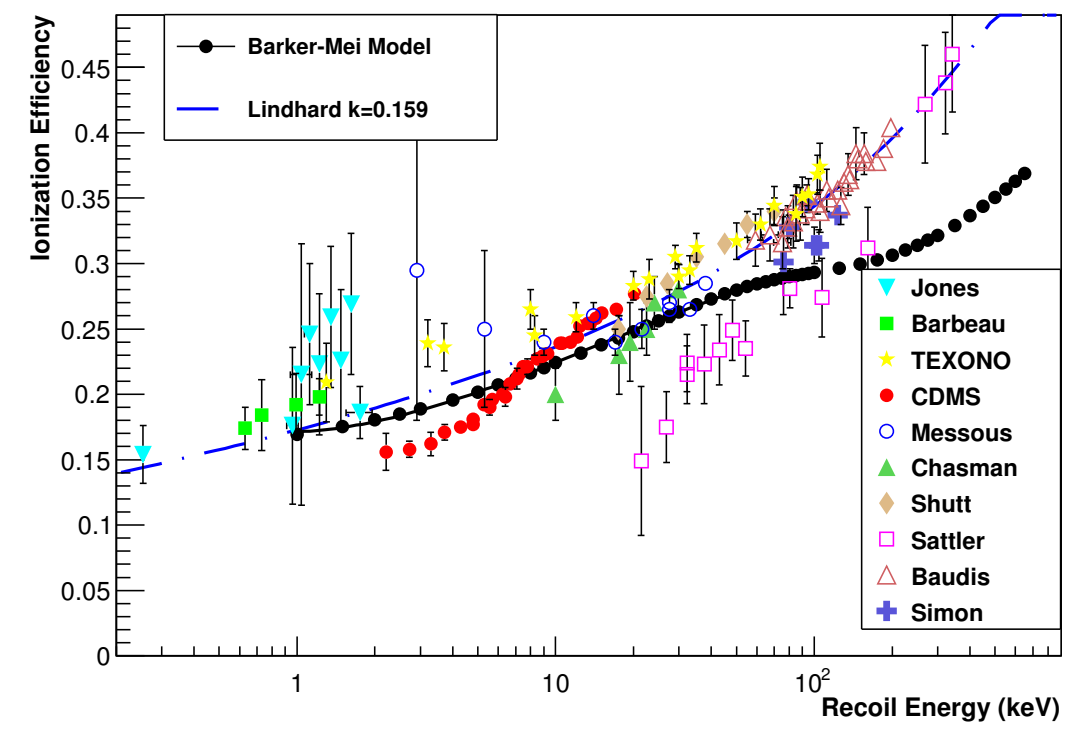

Fig. 1. The Barker-Mei and Lindhard models of ionization efficiency with the experimental data [567789,10/11/12]13].

the germanium detector can contaminate the visible energy. In a measurement of neutron elastic scattering, a Monte Carlo simulation must be incorporated to exclude multiple scatters. It is also necessary to precisely measure the scattering angle and time-of-flight of the out-going neutrons. With inelastic scattering, the Compton scattering due to de-excitation of gamma rays in the detector can contaminate the signal. All of these techniques need to be implemented with an accurate Monte Carlo that reduces systematic errors in order to obtain a reliable ionization efficiency. However, current popular simulation tools often need to be tuned to simulate inelastic scattering processes.

Therefore, we desired a simple method to accurately measure nuclear recoils in germanium. The $E_{0}$ transition of germanium-72 $\left({ }^{72} \mathrm{Ge}\left(\mathrm{n}, \mathrm{n}^{\prime} \mathrm{e}\right)\right)$, which is the internal conversion process for this nucleus, was chosen. The $E_{0}$ transition of ${ }^{72} \mathrm{Ge}\left(\mathrm{n}, \mathrm{n}^{\prime} \mathrm{e}\right)$ is induced when neutrons inelastically scatter off a ${ }^{72} \mathrm{Ge}$ nucleus. After this collision, the ${ }^{72} \mathrm{Ge}$ nucleus is left in an excited state. When the nucleus returns to ground state, it does not directly produce a gamma-ray (as is common for other nuclei), but interacts electromagnetically with the inner shell electrons and causes one to be emitted from the atom [14]. The physics process for the internal conversion of ${ }^{72} \mathrm{Ge}$ can be generalized as:

$$
n+{ }^{72} G e \longrightarrow n^{\prime}+{ }^{72} G e^{*} \hookrightarrow{ }^{72} G e+e^{-}+X-\text { ray }
$$

where ${ }^{72} \mathrm{Ge}^{*}$ denotes the excited $0^{+}$state. The total detectable energy from the ${ }^{72} \mathrm{Ge}^{*}\left(0^{+}\right)$to ${ }^{72} \mathrm{Ge}\left(0^{+}\right)$transition is $691.6 \mathrm{keV}$, which includes energy from the $\mathrm{X}$-ray. This energy is well within the visible range of traditional detectors, 
and both electron and X-ray can be detected with a consistency of approximately $100 \%$. The characteristic energy, $691.6 \mathrm{keV}$, is superimposed with the nuclear recoil of a ${ }^{72} \mathrm{Ge}$ nucleus to form a quasi-triangular shape, which is distinguishable from other processes in the spectrum. Thus, we can observe low energy nuclear recoils without taking low energy nuclear recoil measurements by extrapolating with the known quasi-triangular fit. This quasi-triangular shape, starting at $691.6 \mathrm{keV}$, has been studied in great detail [15,20|21,22,23]. The tail of the quasi-triangular shape comes from a combination of the 691.6 keV energy deposition and nuclear recoil energy due to neutron scattering.

Depending on the incident neutron energy and scattering angle [15], nuclear recoil energy can be expressed as:

$$
E_{r}=\frac{4 m M E_{n}}{(M+m)^{2}}\left(\cos ^{2} \theta\right),
$$

where $\mathrm{E}_{r}$ is the nuclear recoil energy, $\mathrm{E}_{n}$ is the neutron energy, $\mathrm{M}$ is the nucleus mass, $\mathrm{m}$ is the neutron mass, and $\theta$ is the scattering angle between the incident neutron and the recoil nucleus. The quasi-triangular shape is created by adding the energy from the nuclear recoils to the $691.6 \mathrm{keV}$ energy deposition:

$$
E=691.6 \mathrm{keV}+\epsilon \cdot E_{r}
$$

where $E$ is the observed energy and $\epsilon$ is the ionization efficiency. The nuclear recoil energies can be determined using a Monte Carlo simulation with applied ionization efficiency, if an agreement with the measurements is obtained.

To validate the ionization efficiency models proposed by Lindhard and BarkerMei, a Geant4.9.2-based Monte Carlo simulation package [16], corrected for the internal conversion processes, was used. This simulation package was verified using a well-calibrated ${ }^{60} \mathrm{Co}$ radioactive source with the same experimental setup.

Utilizing the spectrum measurements with a substantiated Monte Carlo simulation, we compared the unique quasi-triangular shape induced by the 691.6 $\mathrm{keV}$ electrons and nuclear recoils in the data to the Monte Carlo simulations for the two models. Shape analysis was used to verify the quasi-triangular shape of spectra in the data and Monte Carlo simulations by analyzing data points in the region of interest. We found a good agreement between the measurements and the Lindhard (with $k=0.159$ ) and Barker-Mei models.

In this paper, we corroborate the ionization efficiency models proposed by Lindhard et $a l$ and Barker-Mei with a neutron induced $E_{0}$ transition for ${ }^{72} \mathrm{Ge}$. The experimental design is discussed in Section 2, followed by data analysis in Section 3. The Monte Carlo simulation is demonstrated and validated in 
Section 4, and the comparison with data described in Section 5. Section 6 summarizes our results.

\section{Experimental Design}

The germanium detector used in our experiment was an old coaxial detector from Princeton Gamma Tech, model RG11B/C [17]. Its linearity of energy response between consecutive calibrations was within $0.35 \%$. However, the full width at half maximum (FWHM) was $7.1 \mathrm{keV}$ at the $1173 \mathrm{keV}{ }^{60} \mathrm{Co}$ peak, a factor of two worse in energy resolution than a new germanium detector. Nevertheless, the detector was sufficient for measuring neutron induced internal conversion. We used an ${ }^{241} \mathrm{Am}-{ }^{9} \mathrm{Be}(\mathrm{AmBe})$ source with neutron energies ranging from $\sim 1.0$ to $11.2 \mathrm{MeV}$, at a frequency of $100 \mathrm{~Hz}$ [18].

An AmBe neutron source produces neutrons in four discrete groups: $n_{0}, n_{1}, n_{2}$, and $n_{3}$, which populate the ground state, the $4.443 \mathrm{MeV}$ level, the $7.65 \mathrm{MeV}$ level, and the $9.64 \mathrm{MeV}$ level of the ${ }^{12} \mathrm{C}$ product nucleus, respectively [24,25]. The $n_{1}$ group neutrons, accompanied by $4.443 \mathrm{MeV}$ gamma rays, dominate the production of neutrons in an AmBe source [24,25]. This feature allowed us to set up a coincidence measurement using sodium-iodide (NaI) detectors (Bicron model number 3M3/3 [19]) with a threshold of above $1 \mathrm{MeV}$. Utilizing this coincidence method, the $\mathrm{NaI}$ detectors measured gamma rays while neutrons were detected with the germanium detector. This coincidence pattern required that the $\mathrm{NaI}$ and Ge detectors both trigger in order for an event to be recorded, suppressing random background events generated by gamma rays from surrounding materials. We took approximately 22 days of data with three $\mathrm{NaI}$ detectors and 13 days of data with two NaI detectors.

Due to low neutron emission, the source was placed on the center of the Ge detector cap and held in place by electrical tape. When using three NaI detectors, they were placed on the right, the left, and directly in front of the germanium detector. When using two NaI detectors, they were placed on the right and on the left of the germanium detector. Fig. 2 shows the experimental set-up. The data acquisition was performed using a National Instruments PXI-1031 system [26] and Igor Pro 4.07 software [27]. Each run lasted approximately 4.5 days. The data from each individual run was added consecutively off-line using analysis code from the Root software package [28]. Evidence of the $691.6 \mathrm{keV} E_{0}$ transition peak can be seen in Fig. 3. 

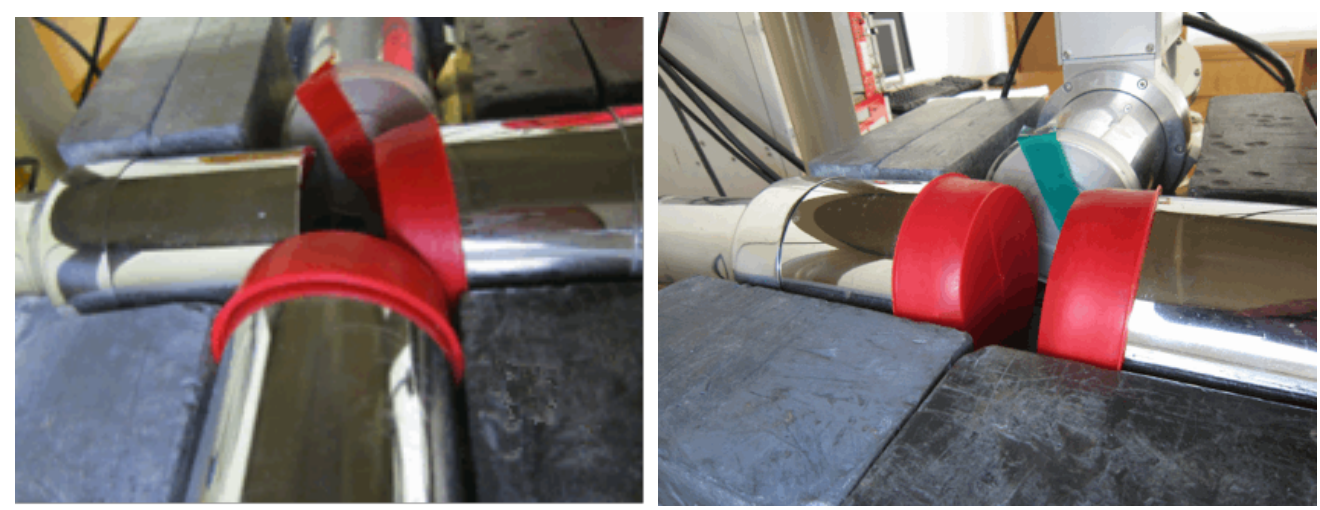

Fig. 2. Experimental setup for Ge and NaI detectors. Left: Three NaI detectors. Right: Two NaI detectors.

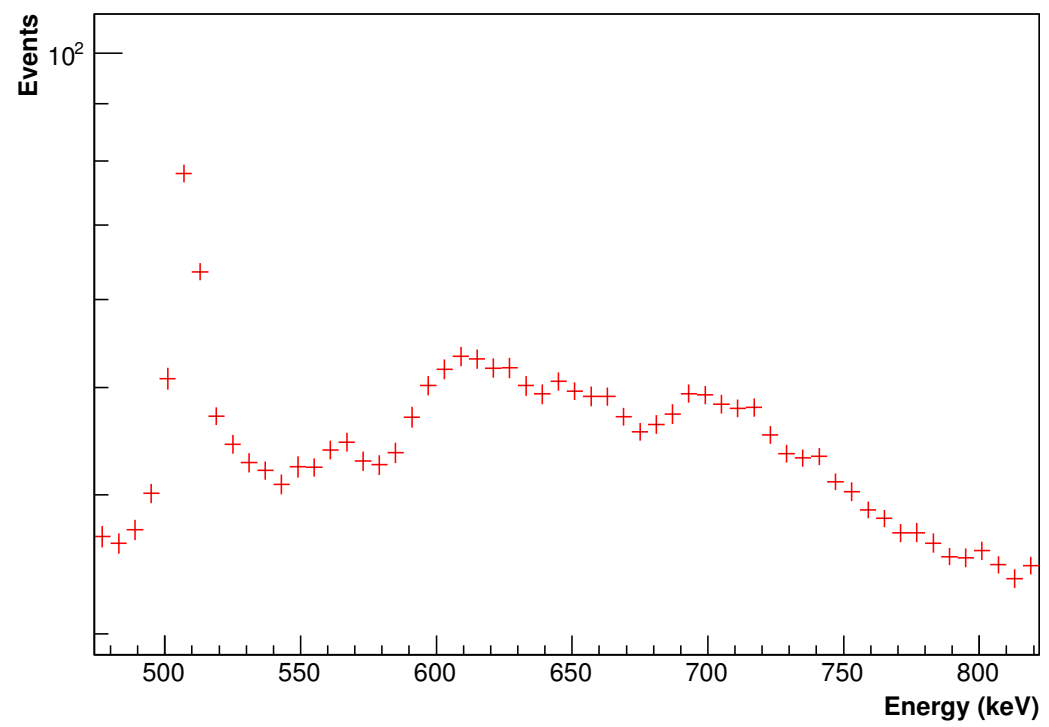

Fig. 3. Data taken with AmBe source after 35 days.

\section{Experimental Results}

\subsection{Data Analysis}

After 35 days, the number of events in the region of interest $(675 \sim 765 \mathrm{keV})$ was approximately 500. This is consistent with the known neutron emission from the AmBe source and the coincidence method, which suppresses random background events from gamma rays, as verified by the Monte Carlo simulation. Because of the small sample size of valid data in the region of interest, the bin size in the histogram was set to $6 \mathrm{keV}$ in order to mitigate statistical fluctuation. As shown in Fig. 3, there are several peaks near the region of interest. We identified these peaks in order to help understand the processes that 
took place within our detector as well as possible sources of contamination.

The first peak can be fitted using a standard Gaussian distribution and linear background distribution. This is given by:

$$
p_{0} \cdot \exp \left[-\frac{1}{2}\left(\frac{E-p_{1}}{p_{2}}\right)^{2}\right]-p_{3}+p_{4} E
$$

where $p_{0}=37 \pm 1.4$ is the normalization constant, $p_{1}=508 \pm 0.2 \mathrm{keV}$ is the center value, $p_{2}=4.7 \pm 0.2 \mathrm{keV}$ is the Gaussian width, $p_{3}=-65 \pm 12$ is a constant, $p_{4}=0.2 \pm 0.02$ is the slope, and $E$ is the energy in keV. The peak at $511 \mathrm{keV}$ is mainly from the annihilation of $\mathrm{e}^{-} \mathrm{e}^{+}$pairs induced by cosmic rays passing through the surrounding materials with other minor contributions. Most notable is the $4.443 \mathrm{MeV}$ induced $\mathrm{e}^{-} \mathrm{e}^{+}$in the surrounding materials. The positrons can annihilate with electrons producing $511 \mathrm{keV}$ gamma rays, which enter the germanium detector. The fitted central value of $508 \mathrm{keV}$ is slightly lower than the expected $511 \mathrm{keV}$, but still within the margin of error for the given bin size.

The remaining peaks can be fitted using a Moyal distribution and linear background. The Moyal distribution used is [29]:

$$
\Psi=\sqrt{\frac{1}{2 \pi} \exp \left[-\left(R\left(E-E_{m p v}\right)+\exp \left[R\left(E-E_{m p v}\right)\right]\right)\right]}
$$

where $R$ is a constant and $E_{m p v}$ is the most probable value of energy deposition in the detector. $R$ and $E_{m p v}$ are physically significant parameters. The value of $R$ is related to the reciprocal of the stopping power and can be used to calculate average path length for particles in the medium. The most probable value of energy, $E_{m p v}$, is the most common energy deposition in that region. These parameters are further discussed in Section 3.3 and used to interpret the experimental data.

The peak around $560 \mathrm{keV}$, is likely caused by events from ${ }^{76} \mathrm{Ge}\left(n, n^{\prime} \gamma\right)$ inelastic scattering. Because of the small number of events, the fitting function was only partially accurate. The second peak at around $600 \mathrm{keV}$ has the fitted parameters, $R=0.06 \pm 0.02$ and $E_{m p v}=609 \pm 3.6 \mathrm{keV}$. This peak is likely the combination of inelastic scattering from ${ }^{74} \mathrm{Ge}\left(n, n^{\prime} \gamma\right)$ and the neutron capture on ${ }^{73} \mathrm{Ge},{ }^{73} \mathrm{Ge}(n, \gamma)$. Finally, the peak of interest, $691.6 \mathrm{keV}$, exhibits the internal conversion of ${ }^{72} \mathrm{Ge}\left(n, n^{\prime} e\right)$. The fitted parameters are, $R=0.06 \pm$ 0.02 and $E_{m p v}=705 \pm 3.0 \mathrm{keV}$. All fits in the region of interest are shown in Fig. 4.

We also took a background spectrum using the coincidence method to identify 


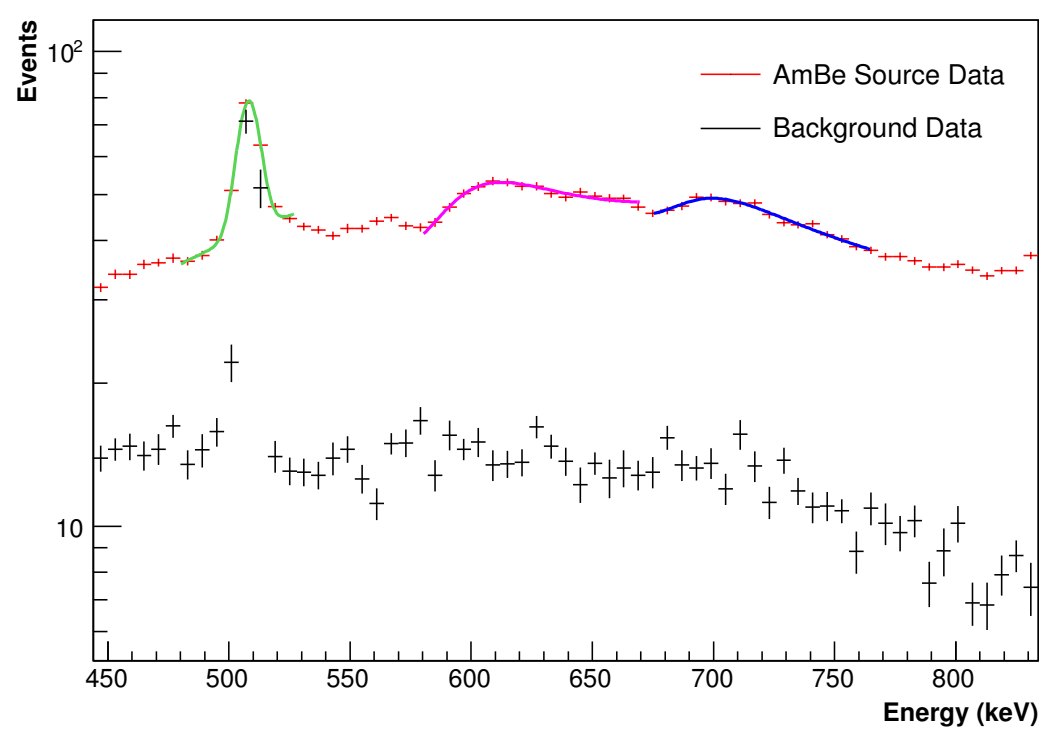

Fig. 4. Region of interest with all peaks fitted, and the coincidence background spectrum.

any random coincidence. This spectrum is also shown in Fig. 4. The background data was taken using two sodium iodide detectors (see right of Fig. 2) for 4.6 days.

After the background spectrum was collected, we subtracted it from the AmBe data sets as shown by Fig. 5. The $511 \mathrm{keV}$ peak was significantly reduced, veri-

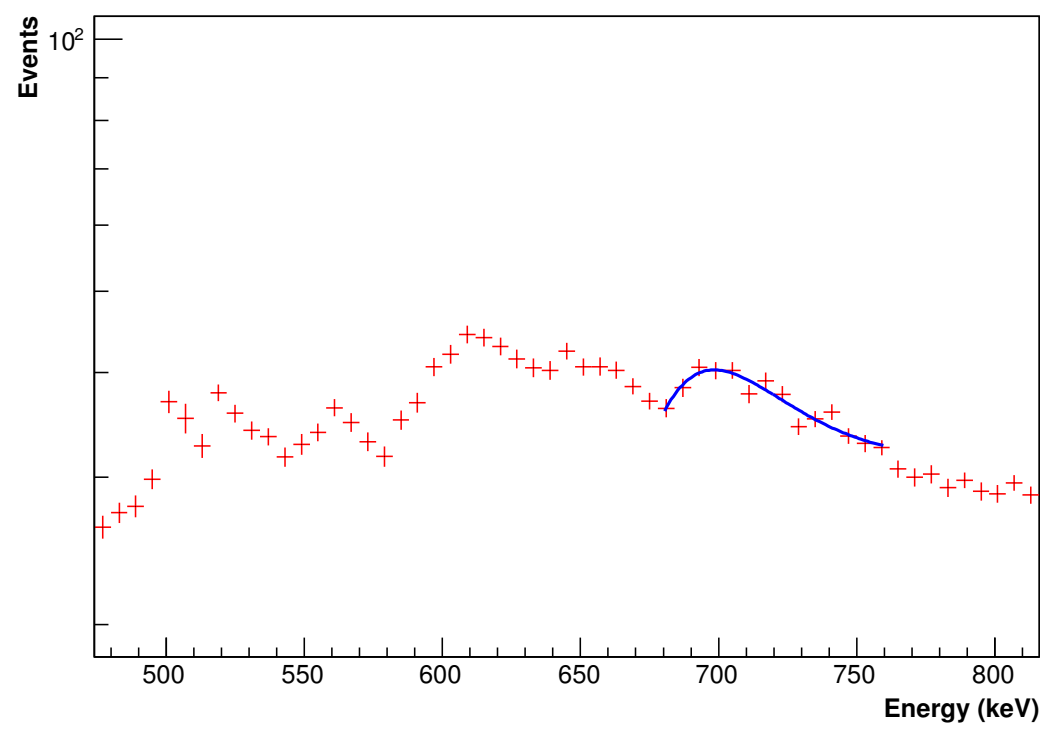

Fig. 5. Region of interest with the background subtracted from the original data files. 
fying our conjecture on the main origin of this peak as induced pair production from cosmic rays in the surrounding materials. The subtracted data set was also fitted using a Moyal distribution and linear background. It was found that the most probable value of energy deposition in the detector for the $E_{0}$ transition was $696 \pm 1.4 \mathrm{keV}$. Thus the percent difference of the AmBe data with and without background subtraction is $1.3 \%$.

\subsection{Error Analysis}

We have identified the following sources of systematic error for our data analysis: the energy scale and the energy resolution. The associated energy scale error is $3 \mathrm{keV}$, which is from the fit of the known $511 \mathrm{keV}$ peak. The center value of the fitted function was $508 \mathrm{keV}$ which is a difference of $3 \mathrm{keV}$ from the known value of the peak. This shift in energy is from the energy calibration used to convert to the energy scale. As stated previously, there was a $0.35 \%$ error in successive energy calibrations. Using a more accurate energy scale to produce a peak at $511 \mathrm{keV}$ caused other energy regions to become less accurate. For energy resolution, the value from the $662 \mathrm{keV}$ peak of ${ }^{137} \mathrm{Cs}$ was used, it is nearest of the calibration sources to our region of interest. The energy resolution for this peak was $3.45 \mathrm{keV}$. Adding two errors in quadrature, since they are independent of each other, causing the resulting systematic error to be $4.6 \mathrm{keV}$. Thus, the larger bin size of $6 \mathrm{keV}$ was used to accommodate for this error.

Statistically, there are approximately 500 events in the region of interest (675 $\mathrm{keV} \sim 765 \mathrm{keV})$ which gives a statistical error of $4.5 \%$. For data with the background subtracted out, the error associated with the most probable value is $696 \pm 4.6$ (sys) \pm 1.4 (stat).

\subsection{Interpretation of Results}

Electrons and nuclear recoils that travel through germanium with a high momentum lose energy by exciting and ionizing the germanium atoms. The average amount of energy lost can be calculated with the Bethe-Bloch equation [30. However, the energy transfer is not a continuous process. It occurs through random collisions during which various amounts of energy can be transferred. The energy loss in the detector can be described by the Moyal distribution, Eq. 7, as shown in Fig. 4.

As shown in Eq.7, $R$ is the reciprocal of the density, $\rho$, times the average path-length of charged particles in the detector, $d$, times the parameter, $K$, 
as given by the Bethe-Block formula:

$$
R=\frac{1}{K \cdot \rho \cdot d}
$$

where $K=4 \pi N_{a} m_{e} c^{2} r_{e}^{2} z^{2} \frac{Z}{A} \frac{1}{\beta^{2}}$ is related to the stopping power, with constants $N_{a}$, Avogadro's number; $m_{e}$, the mass of electrons; $c$, the speed of light; $r_{e}$, the Bohr radius; $z$, the electron charge; $Z$, atomic number of target; $A$, the atomic mass number of target; and $\beta$, the speed of charged particles divided by $c$. The density of germanium is $\rho=5.323 \mathrm{~g} / \mathrm{cm}^{3}$.

Since electronic stopping power is different from nuclear stopping power, the value of $R$ is very different for pure electronic recoils than nuclear recoils. Thus, the value of $R$ can be used to derive the average path-length for a given electronic recoil or nuclear recoil. However, the value of $R$ from our measurements is a combination of electronic and nuclear recoils, and it cannot be used to directly determine the average path length for either. Nevertheless, we can use the most probable value of energy deposition, which is related to the stopping power multiplied by the average path length, to determine the average path length.

Given $E_{m p v}=696 \pm 4.6$ (sys) \pm 1.4 (stat) keV, obtained from the fitted function in Fig. 5, is a convolution of the $691.6 \mathrm{keV}$ energy deposition and the nuclear recoils, then we contend that $696 \mathrm{keV}=\frac{d E}{d X} \cdot d \cdot \rho$, where $\frac{d E}{d X}$ is the mass stopping power in $\mathrm{keV} \mathrm{cm}^{2} / \mathrm{g}$. Since there are both electronic and nuclear recoils, we can rewrite this as:

$$
696 \pm 4.6 \pm 1.4 k e V=\left(\frac{d E}{d X_{e}} \cdot d_{e}+\frac{d E}{d X_{n}} \cdot d_{n}\right) \cdot \rho
$$

where $\frac{d E}{d X_{e}}=1301 \mathrm{keV} \mathrm{cm}{ }^{2} / \mathrm{g}$ [31] and $\frac{d E}{d X_{e}} \cdot d_{e} \cdot \rho=691.6 \mathrm{keV}$; thus $d_{e}=$ $691.6 /\left(\frac{d E}{d X_{e}} \cdot \rho\right)=0.1 \mathrm{~cm}$, which is the average path length in germanium for electrons with an energy of $691.6 \mathrm{keV}$. Therefore, we have

$$
696 \pm 4.7 \pm 1.4 \mathrm{keV}=691.6 \mathrm{keV}+\frac{d E}{d X_{n}} \cdot d_{n} \cdot \rho
$$

and

$$
4.4 \pm 0.007 \pm 0.002 \mathrm{keV}=\frac{d E}{d X_{n}} \cdot d_{n} \cdot \rho .
$$

From Eq.11, we can conclude that the most probable nuclear recoils that we measured in the detector have an average of $4.4 \pm 0.007$ (sys) \pm 0.002 (stat) 
$\mathrm{keV}$ electronic equivalent energy, which corresponds to approximately $17.5 \pm$ 0.12 (sys) \pm 0.035 (stat) keV nuclear recoil energy from ionization efficiency in the Barker-Mei model.

To determine $d_{n}$, we can use $\frac{d E}{d X_{n}}=2341720 \mathrm{keV} \mathrm{cm} / \mathrm{g}$ for a nuclear recoil of $17.5 \mathrm{keV}$ [4]. Thus, $d_{n}=17.5 \mathrm{keV} /\left(\frac{d E}{d X_{n}} \cdot \rho\right)=0.014 \mu \mathrm{m}$ is the average path length in germanium for $17.5 \mathrm{keV}$ nuclear recoils. This determines the range of low energy nuclear recoils in a germanium detector.

\section{Monte Carlo Simulation of $E_{0}$}

An accurate Monte Carlo simulation is needed to determine the validity of the Lindhard and Barker-Mei models when compared to collected data. Two crucial steps were taken before creating a Monte Carlo simulation that would determine nuclear recoil energy: 1) the $E_{0}$ transition in Geant4.9.2 [16] was fixed and 2) the Monte Carlo simulation was verified with a well-known gamma-ray source.

\subsection{Fixing the $E_{0}$ transition in Geant4.9.2}

By studying the inelastic scattering processes that contribute to internal conversion, we found that the $E_{0}$ transition code is included in Geant4, but is missing neutron data for ${ }^{72}$ Ge. Specifically, Geant4 does not provide $\gamma / e$ transition data in each energy level (Data Type $=12$ ) and has no cross section data corresponding to the $E_{0}$ transition.

In order to make the internal conversion process available in our Geant4 simulation, we created a $\gamma / e$ ratio in the database for ${ }^{72} \mathrm{Ge}$ with Geant4.9.2 several years ago (see our correction in Ref. [32]). In addition, we calculated all the cross-sections of the ${ }^{72} \mathrm{Ge}\left(n, n^{\prime}\right)$ reaction using TALYS [33], a reliable software for the simulation of nuclear reactions. We then converted the transition cross-section data into the transition ratio in Geant4.9.2 as shown in Fig. 6 (we utilize Geant4.9.2 for this work because the $\mathrm{E}_{0}$ transition problem was amended several years ago).

The independence of this ratio can be cross-checked by looking at the reactions in different energy scales. Eventually, the TALYS data was converted to Geant4 format where it could be used in the internal conversion process (see the supplemental data used in Geant4.9.2 from Ref. [32]).

During the $E_{0}$ transition, a characteristic X-ray, or Auger electron, is produced 


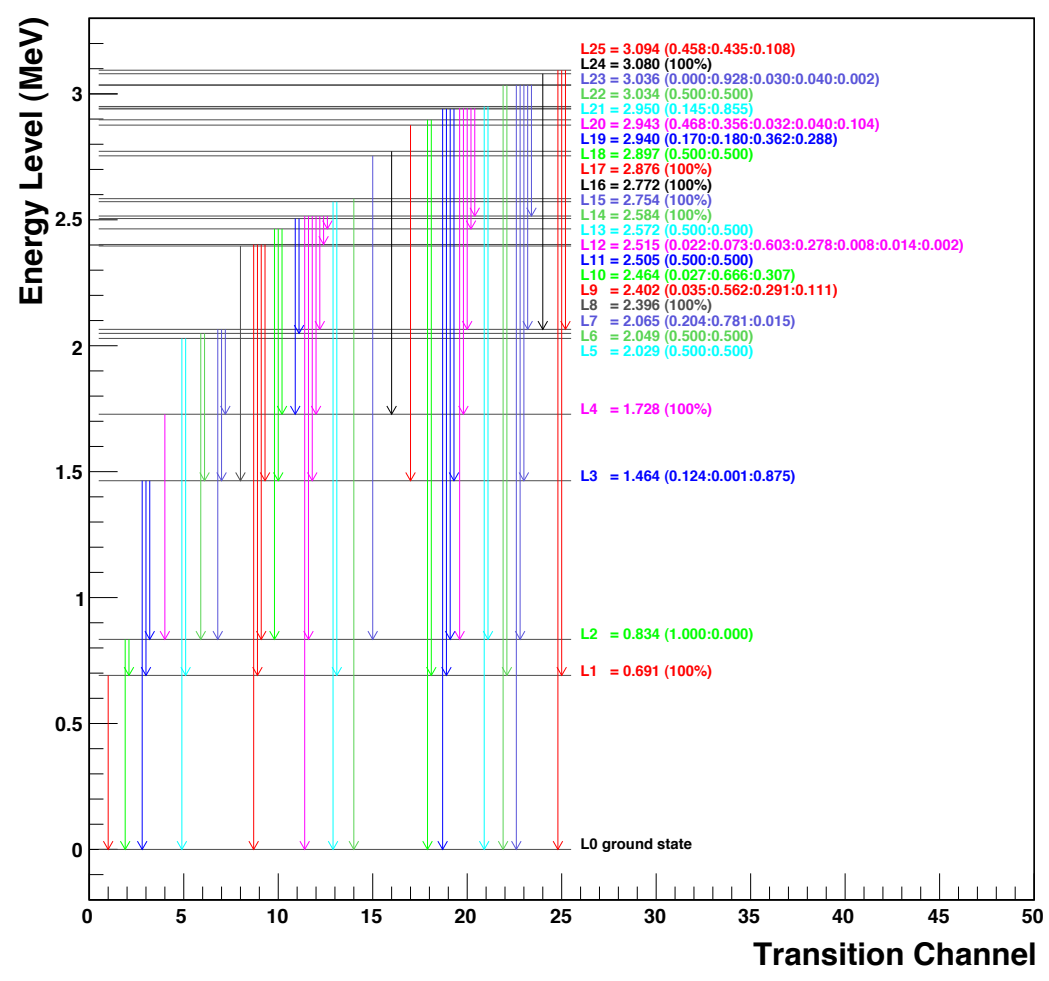

Fig. 6. The orbital $e^{-}$transition ratio of ${ }^{72} \mathrm{Ge}$ in its excited states.

simultaneously with the conversion electron. This process was not included in the Geant4 code. However, since this is a complicated process that involves binding energy, it would require editing for all the elements in the Geant4 package. For the sake of simplicity, we combined the X-ray and conversion electron together, since they both contribute to the total energy deposition (in a solid germanium detector only). A more general solution will be required when considering other materials.

A simulation was created from the Geant4.9.2 and G4NDL3.13 packages that includes the additional transition ratio data. For further information, readers can refer to Ref. [37] for detailed geometry and experimental framework. After the missing data was added, the $E_{0}$ transition of ${ }^{72} \mathrm{Ge}\left(n, n^{\prime} e\right)$ could be simulated using Geant4.9.2. This makes the Geant4.9.2 simulation more reliable for dark matter searches utilizing germanium detectors.

\subsection{Verification of the Monte Carlo Simulation}

To obtain reliable results from our Monte Carlo, it was essential to verify the simulation. ${ }^{60} \mathrm{Co}$, with an original radioactivity of $1.0 \mu \mathrm{Ci}$, was used to 

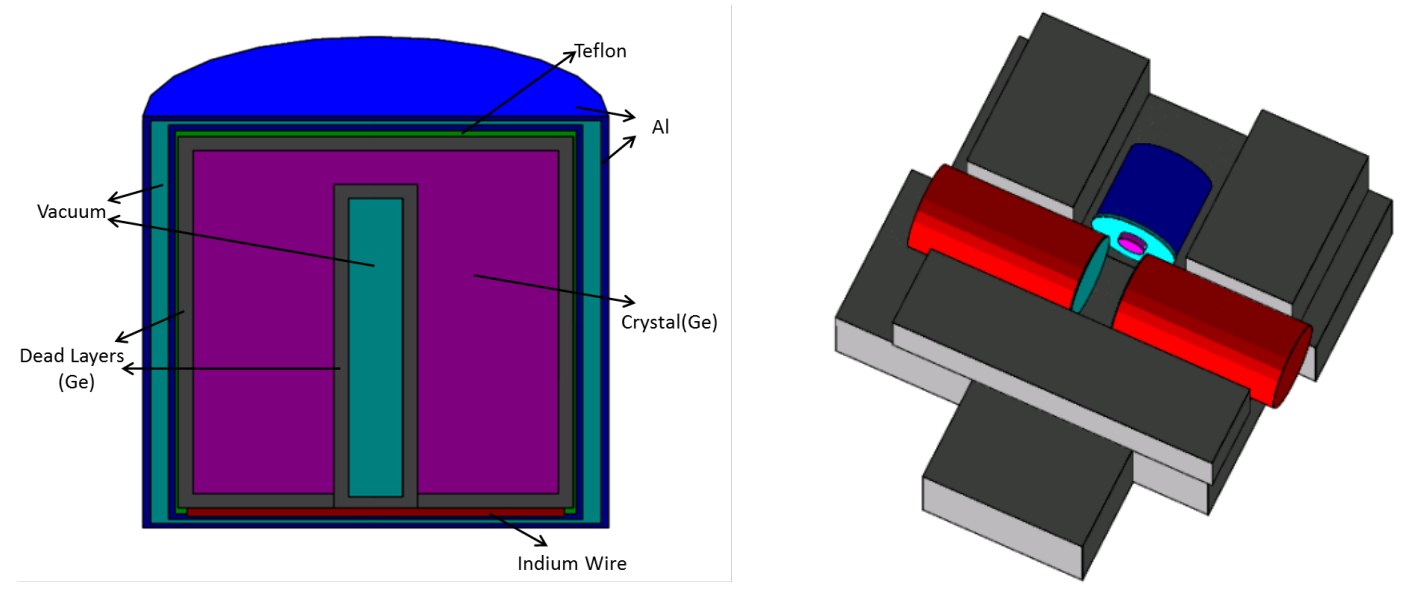

Fig. 7. Simulated geometry for Ge and NaI detectors. Left: Cross section of Ge detector. Right: Ge detector (Blue), two NaI detectors (Red) and Lead bricks (Gray). validate the simulation. The ${ }^{60} \mathrm{Co}$ source was mounted on the center of the Ge detector cap as shown in Fig. 2 (right). The corresponding geometry used in the Monte Carlo simulation is shown in Fig. 7. Since the germanium detector is aged, the Monte Carlo geometry was modified to include a dead layer of thickness $2.5 \mathrm{~mm}$ (see Fig. 7, left). We also implemented a smearing process by applying energy resolution to the spectrum of deposited energy in the active germanium. When fitting the energy resolution, these four peaks were identified: $1173 \mathrm{keV}, 1332 \mathrm{keV}, 2506 \mathrm{keV}$ and $662 \mathrm{keV}$. The first three peaks are gamma rays from the ${ }^{60} \mathrm{Co}$ source and the last peak is a gamma ray from ${ }^{137} \mathrm{Cs}$ source. The best fit function is presented in Eq. 12 .

$$
0.2017 \pm 0.00846 \times \sqrt{0.3+(0.001 E)^{(-1.96 \pm 1.69)}+(0.001 E)^{(-3.436 \pm 1.262)}},
$$

where $E$ is the energy in $\mathrm{keV}$. Under the square root, the first term, 0.3 , is the percentage of energy resolution, for a new Ge detector; the second and third terms are due to noise and the age of the Ge detector. The percentage of relative energy resolution as a function of energy is plotted in Fig. 8 Using this fitted function, we applied the energy resolution to the range $600 \mathrm{keV} \sim$ $2510 \mathrm{keV}$ and obtained agreement between the experimental data and Monte Carlo simulation as shown in Fig. 9, This validates that our Monte Carlo can be reliably used for gamma-ray simulation.

\section{Monte Carlo Simulation of Nuclear Recoils}

Using the modified Geant4.9.2 package, we performed simulations to verify the accuracy of the Lindhard and Barker-Mei models with an AmBe neutron 


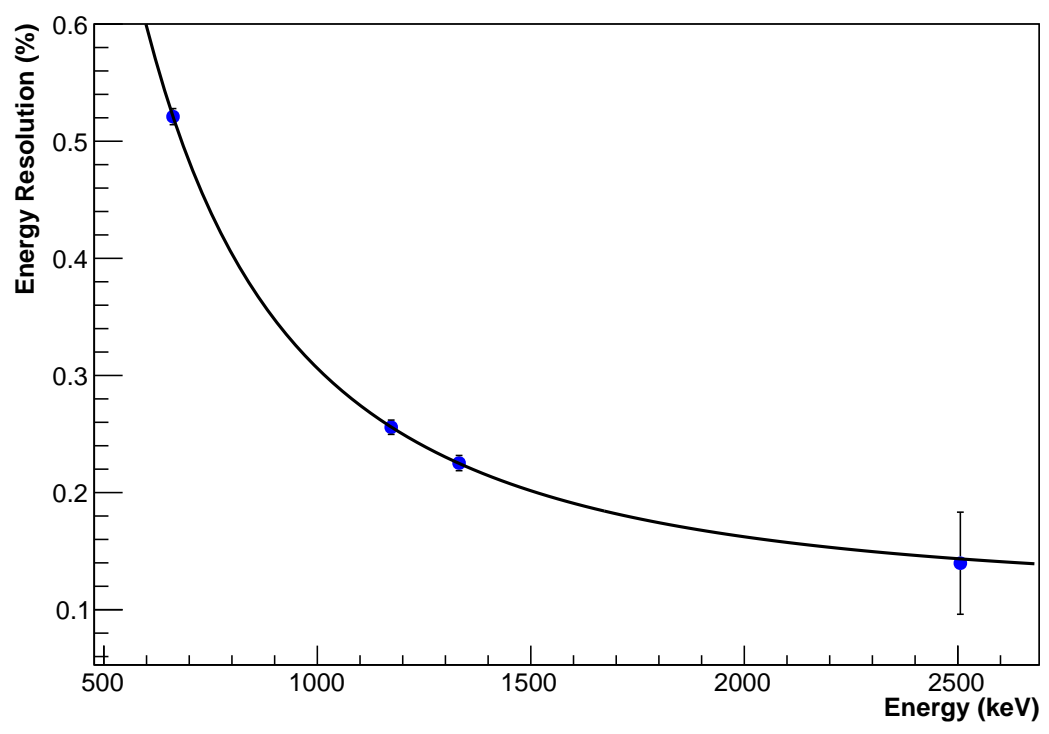

Fig. 8. Percentage of relative energy resolution as a function of energy. The error bars are statistical errors.

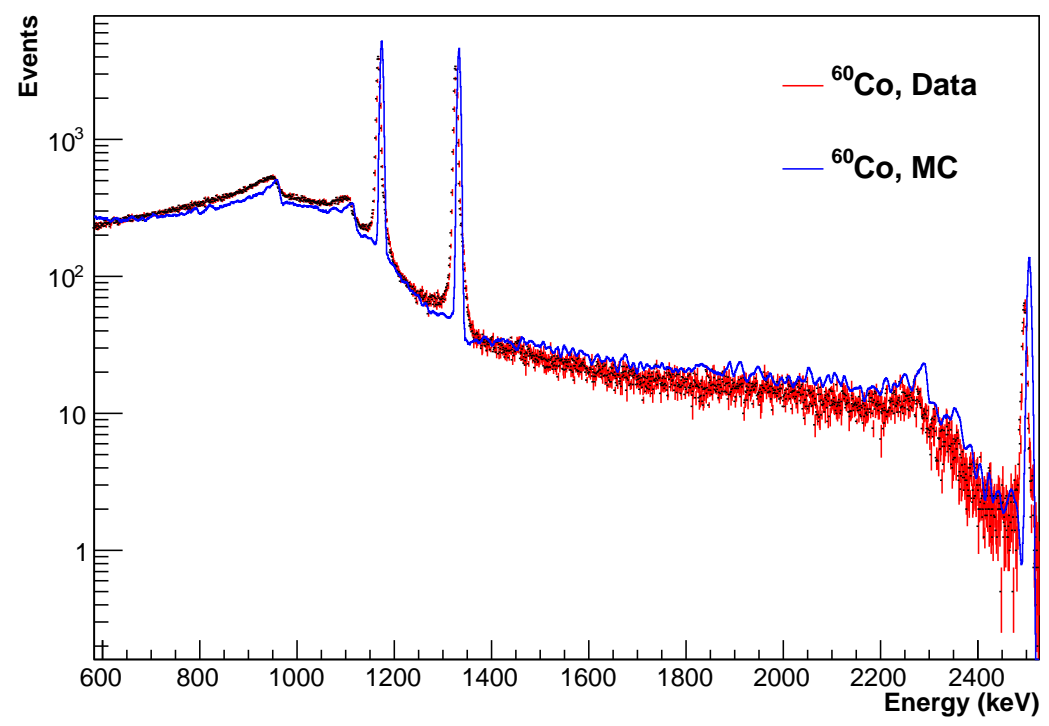

Fig. 9. Comparison between MC and data in energy deposition spectrum.

source. The experimental setup for our Ge and NaI detectors, as shown in Fig. 2, was simulated according to the dimension and material information provided by the manufacturer [34]. Since the ${ }^{72} \mathrm{Ge}\left(n, n^{\prime} e\right)$ reaction causes a quasi-triangular shape in the data, we expect to see this same feature in our Monte Carlo simulation if the Lindhard and Barker-Mei models are accurate. In order to provide an accurate simulation, the geometry, as well as the AmBe source generator, need to be implemented correctly. 


\subsection{AmBe Neutron Generator}

Because the AmBe source was placed very close to the germanium detector, gamma rays emitted from the source have a greater chance of entering the germanium detector and causing contamination in the region of interest (675 $\mathrm{keV} \sim 765 \mathrm{keV})$. Thus, in the simulation it is necessary to account for all potential gamma rays emitted from the AmBe source.

Two reactions, shown in Eqs. 13 and 14, occur in the AmBe source:

$$
\begin{aligned}
& { }^{241} \mathrm{Am} \rightarrow{ }^{237} \mathrm{~Np}+\alpha+\gamma \\
& \alpha+{ }^{9} \mathrm{Be} \rightarrow{ }^{12} \mathrm{C}+n+\gamma
\end{aligned}
$$

Eq. 13 shows the decay of ${ }^{241} \mathrm{Am}$ to ${ }^{237} \mathrm{~Np}$, which causes the emission of alpha particles and gamma rays. Eq. 14 shows the reaction between an alpha particle and ${ }^{9} \mathrm{Be}$. In Eq. 14, the energy of gamma rays emitted depends on the resulting state of ${ }^{12} \mathrm{C}$, which is $4.443 \mathrm{MeV}$ (for the 1 st excited state), $7.65 \mathrm{MeV}$ (for the 2nd excited state) and $9.64 \mathrm{MeV}$ (for the third excited state). Using the gamma ray energy and recoil energy of ${ }^{12} \mathrm{C}$, the resulting neutron energy can be calculated by applying energy conservation to Eq. 14 .

Fully absorbed gamma rays (from Eq. 14) will not be in the region of interest $(675 \mathrm{keV} \sim 765 \mathrm{keV})$ since their energies are at a few $\mathrm{MeV}$. However, the Compton continuum of their interaction can contribute to the region of interest. In addition, gamma rays (from Eq. 13) with energies $26.34 \mathrm{keV}$ (a branching ratio of $2.4 \%$ ), $59.54 \mathrm{keV}$ (a branching ratio of $35.9 \%$ ) and 722.01 $\mathrm{keV}$ (a branching ratio of $0.000196 \%$ ) [35] can contribute to the region of interest by occurring in coincidence with the $\mathrm{E}_{0}$ transition $(26.34 \mathrm{keV}$ and 59.54 $\mathrm{keV})$ or by becoming fully absorbed $(722.01 \mathrm{keV})$. We generated these three gamma rays in our Monte Carlo simulation along with gamma rays at energies of $4.443 \mathrm{MeV}, 7.65 \mathrm{MeV}$, and $9.64 \mathrm{MeV}$ caused by the transitions in the excited ${ }^{12} \mathrm{C}$ nucleus.

The simulated neutron energy spectrum from the AmBe neutron source is shown in Fig. 10. The spectrum agrees with the prediction from Marsh et. al. [36]. After this validation, the AmBe source generator was implemented in the Monte Carlo simulation.

\subsection{Verification of the $E_{0}$ Transition in Simulation}

The simulated results are presented as a spectrum of energy deposited in the germanium detector after the application of smearing, which is the process of 


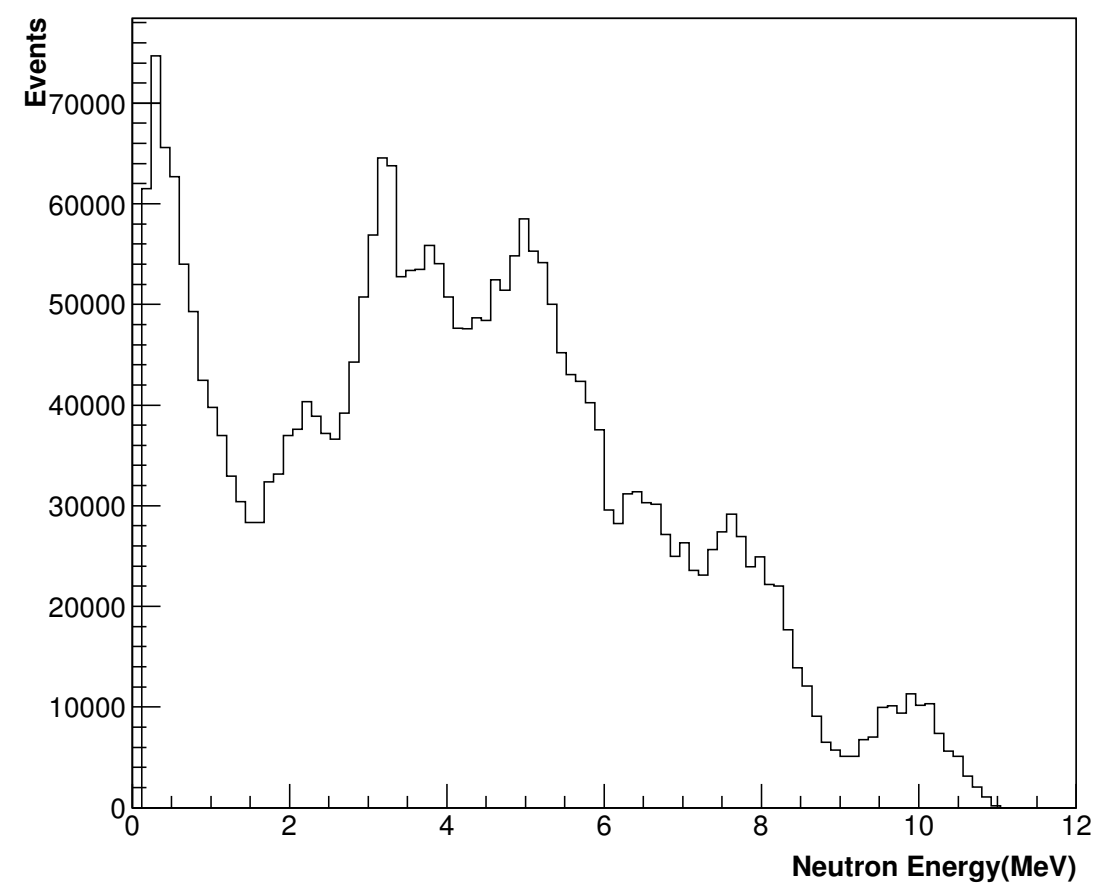

Fig. 10. Simulated neutron energy spectrum from AmBe neutron source.

accounting for energy resolution in a germanium detector.

Gaussian and Moyal distributions with fitted parameters from our data (Section 3) have been incorporated into the smearing of different peaks in the region of interest. We used the model proposed by Lindhard et al. 3 . $(k=$ $0.159)$ to determine the ionization efficiency for a germanium detector. Fig. 11 shows the simulated energy deposition spectrum after smearing. The $E_{0}, 691.6$ $\mathrm{keV}$, transition is visible, as shown in Fig. 11. Appearance of this peak indicates that the internal conversion process has been successfully implemented in our Geant4.9.2.

\section{Monte Carlo Shape Analysis}

After successfully simulating the AmBe neutrons in our Geant4.9.2, we collected the energy deposited in the germanium detector. This collected energy spectrum was compared with our data after the application of smearing (Eq. 12). Two models utilizing ionization efficiency functions were applied to the Monte Carlo spectrum: Lindhard $k=0.159$ and the Barker-Mei model. Normalization was applied to the energy range 675 to $765 \mathrm{keV}$. By overlaying the Monte Carlo energy spectrum and the collected data, we were able to perform a shape analysis on the characteristic $E_{0}$ transition. This is demonstrated 


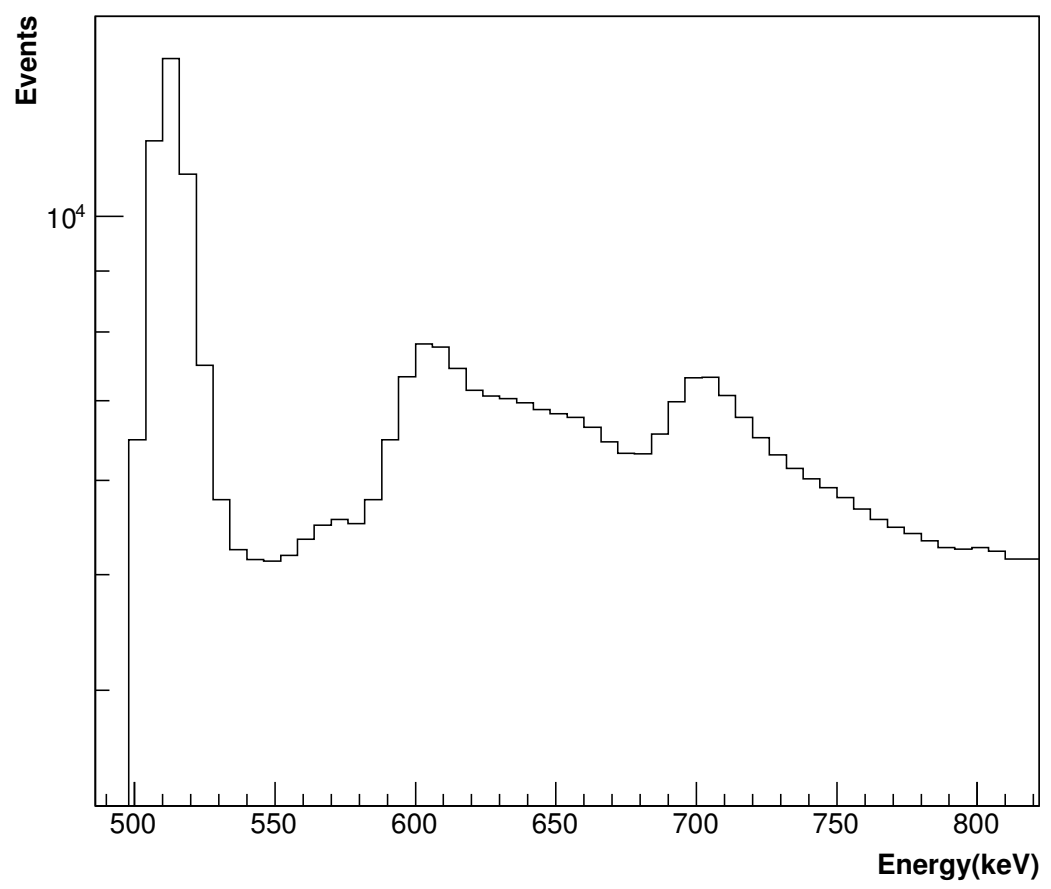

Fig. 11. The spectrum of energy deposition in the germanium detector after smearing.

Table 1

The percent difference between the collected data and the Monte Carlo simulations with two models.

\begin{tabular}{|c|c|c|c|c|c|c|c|c|c|}
\hline \hline Energy $(\mathrm{keV})$ & 691.6 & 696 & 702 & 708 & 714 & 720 & 726 & 732 & 738 \\
\hline Lindhard (k=0.159) (\%) & 2.8 & 1.4 & 3.9 & 3.1 & 1.0 & 0.8 & 2.1 & 2.8 & 3.0 \\
\hline Barker-Mei (\%) & 2.6 & 1.0 & 3.2 & 2.3 & 0.6 & 0.9 & 1.8 & 2.2 & 2.1 \\
\hline \hline
\end{tabular}

in Fig. 12 .

The shape analysis was performed on a bin-to-bin basis by comparing the data and two Monte Carlo simulations, which correspond to the two models. The difference in shape between the data and the two Monte Carlo simulations was calculated using $\frac{(\text { data-MC) }}{(\text { data }+M C) / 2}$. Table 1 shows the results of this comparison in which the largest difference is shown to be less than $4 \%$.

Unfortunately, we were not able to include the neutron capture lines of some specific even nuclei, such as ${ }^{70} \mathrm{Ge}$, in our Geant4.9.2 package due to the lack of adequate cross-sections. Thus, at $708.2 \mathrm{keV}$ and $747.7 \mathrm{keV}$, there is a discrepancy between the collected data and Monte Carlo simulations due to the neutron capture of ${ }^{70} \mathrm{Ge}(n, \gamma)$ [37. There is also inconsistency in the energy range $650 \sim 680 \mathrm{keV}$ that is likely due to ${ }^{115} \operatorname{In}(n, \gamma)$ and ${ }^{206} \mathrm{~Pb}\left(n, n^{\prime} \gamma\right)$ [37] 


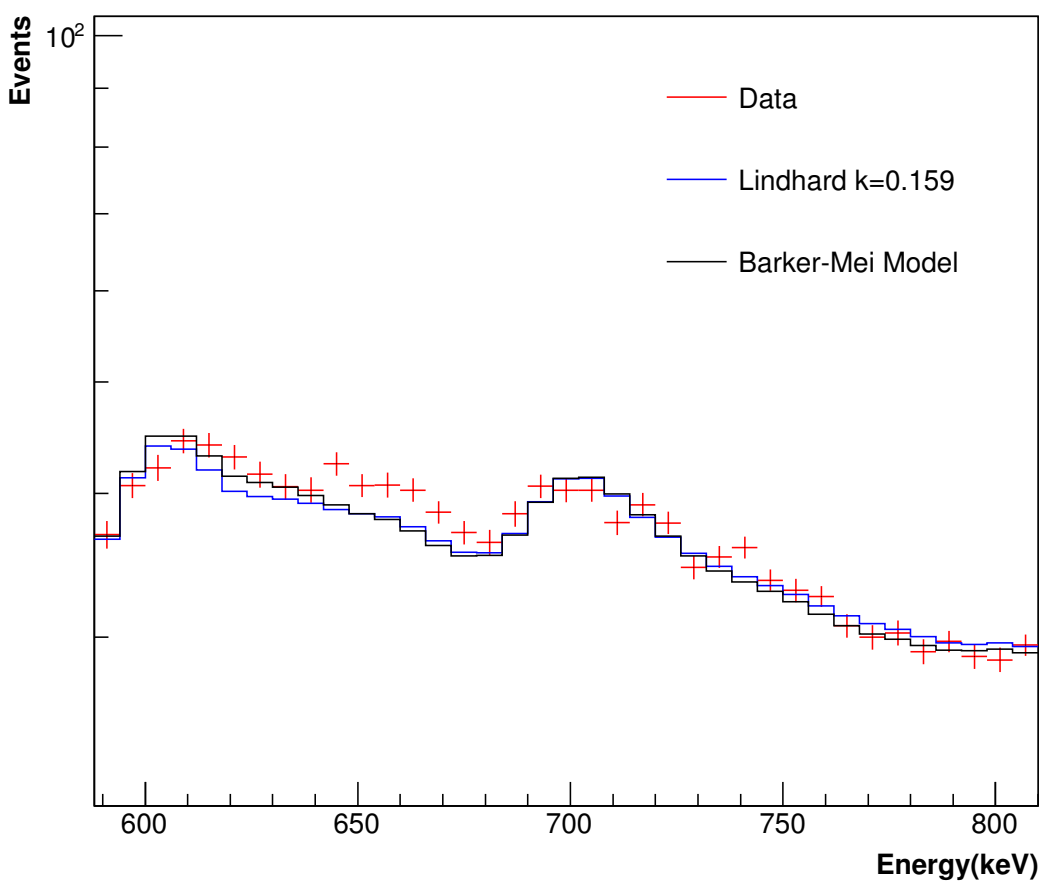

Fig. 12. Comparison of our Monte Carlo simulation utilizing the Lindhard $(k=$ 0.159) and Barker-Mei models and our collected data after background subtraction. The Monte Carlo simulation is normalized to the experimental live-time.

Table 2

The extracted average visible nuclear recoil energy from the data and the corresponding nuclear recoil energy from the Monte Carlo simulation. $\mathrm{E}_{v r}$ contains a statistical error of $14 \%$ per energy bin and a systematic error of $4.6 \mathrm{keV}$ added in quadrature. There are no errors assigned to the nuclear recoil energy obtained from the Monte Carlo simulation.

\begin{tabular}{|c|c|c|c|c|c|c|c|}
\hline \hline $\mathrm{E}_{v r}(\mathrm{keV})$ & $0.5 \pm 0.5$ & $1.4 \pm 1.4$ & $4.4 \pm 4.4$ & $10.4 \pm 4.8$ & $16.4 \pm 5.1$ & $22.4 \pm 5.6$ & $28.4 \pm 6.1$ \\
\hline $\mathrm{E}_{r}(\mathrm{keV})$ & 2.7 & 6.7 & 17.7 & 37.3 & 56.6 & 76.3 & 97.3 \\
\hline \hline
\end{tabular}

processes, which are not included in the Geant4.9.2 package.

Since a good agreement between the data and two models was achieved in the shape analysis, we extracted an average visible nuclear recoil energy, $\mathrm{E}_{v r}$, from each bin using the difference between the measured visible energy and the ${ }^{72} \mathrm{Ge}^{*}\left(0^{+}\right)$to ${ }^{72} \mathrm{Ge}\left(0^{+}\right)$transition energy, $691.6 \mathrm{keV}$. The corresponding nuclear recoil energy, $\mathrm{E}_{r}$, was determined utilizing the Monte Carlo simulation. Table 2 displays the extracted results.

Using $\frac{E_{v r}}{E_{r}}$, Fig. 13 shows the extracted ionization efficiency from the data and Monte Carlo simulation. Note that this is not a direct measurement of ionization efficiency, but an extraction using the shape analysis. 


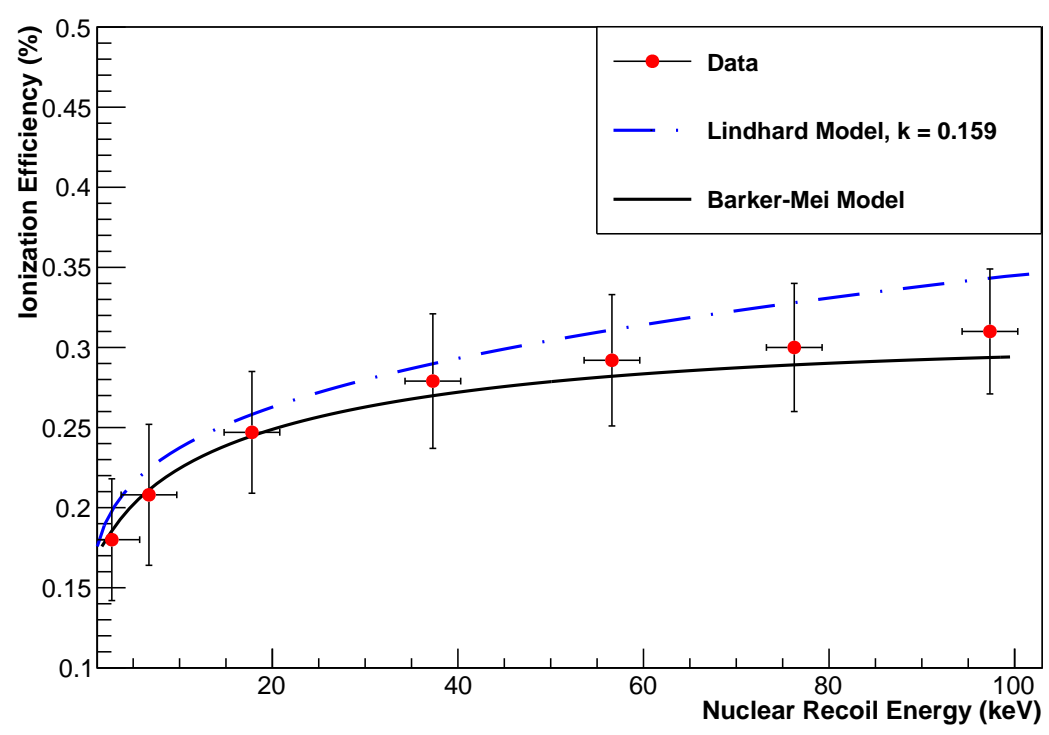

Fig. 13. Extracted ionization efficiency from the shape analysis. The error bars account for a statistic error of $14 \%$ per bin and the variation due to the systematic error of $4.6 \mathrm{keV}$ added in quadrature.

\section{Conclusion}

We took measurements using an AmBe neutron source, incident on a germanium detector, for a total of 35 days. The characteristic quasi-triangular shape located at $691.6 \mathrm{keV}$ represents the $\mathrm{E}_{0}$ transition of germanium-72. All peaks in the region of interest have been identified, thereby confirming source related and environmental backgrounds. The unique quasi-triangular shape induced by neutrons can be described using the Moyal distribution with the fitted parameters $R=0.06 \pm 0.01$ (stat) and $E_{m p v}=696 \pm 4.6$ (sys) \pm 1.4 (stat) keV, after background subtraction. Utilizing these parameters, we derived the most probable value for nuclear recoils as $4.4 \pm 0.007$ (sys) \pm 0.002 (stat) keV electronic equivalent energy, which corresponds to a nuclear recoil energy of $17.5 \pm 0.12$ (sys) \pm 0.035 (stat) keV. The average path length in the germanium detector for $17.5 \mathrm{keV}$ nuclear recoils was approximately 0.014 $\mu \mathrm{m}$.

A Monte Carlo simulation employing a corrected Geant4.9.2 package was modified to duplicate the same experimental setup and AmBe neutron source. The Lindhard $(k=0.159)$ and Barker-Mei models were used to apply ionization efficiency to the energy spectrum and were compared to the experimental measurements. A bin-to-bin shape analysis was performed, and the difference between the measurements and two models were calculated. We obtained a percent difference that was less than $4 \%$ for the collected data and two Monte Carlo simulations (see Table 1). Using the shape analysis, we calculated the 
most probable values for visible nuclear recoils (as shown in Table 1), extracted the average visible nuclear recoil energy (see Table 2), and obtained the corresponding nuclear recoil energy from the Monte Carlo simulation (see Table 2). The extracted average ionization efficiency is shown in Fig. 13. These values are in agreement with the Lindhard and Barker-Mei models in the energy range of 1 to $100 \mathrm{keV}$. Therefore, the Lindhard model (with $k=0.159$ ) and Barker-Mei model can be used to determine the ionization efficiency in germanium detectors for 1 to $100 \mathrm{keV}$ nuclear recoil energy.

\section{Acknowledgments}

The authors wish to thank Iseley Marshall and Angela A. Chiller for their careful reading of this manuscript. Additionally, the authors would like to thank Rupak Mahapatra for his comments and suggestions. This work was supported in part by NSF PHY-0758120, DOE grant DE-FG02-10ER46709, the Office of Research at the University of South Dakota and a 2010 research center support by the State of South Dakota.

\section{References}

[1] C.E. Aalseth et. al. (CoGeNT), Phys. Rev. Lett. 107, 141301 (2011).

[2] Z. Ahmed et. al. (CDMS), arXiv:1203.1309 (2012).

[3] J. Lindhard et. al., Mat. Fys. Medd. K. Dan. Vidensk. Selsk. 33, 1 (1963).

[4] D. Barker and D.-M. Mei, Astroparticle Physics, Volume 38, p. 1-6. arXiv:1203.4620v4.

[5] K.W. Jones and H.W. Kraner Phys. Rev. C, 4125 (1971).

[6] P.S. Barbeau, J.I. Collar and O. Tench JCAP09(2007)009; P. Barbeau, PhD. Thesis, U. Chicago (2009).

[7] Xichao Ruan, Nuclear recoil quenching factor measurment for HPGe detector (CDEX-TEXONO Collaboration), Beijing, China, 24-26 March 2011.

[8] Y. Messous et. al. Astrophys. 3 (1995) 361-366.

[9] C. Chasman et. al. Phys. Rev. Lett., 211430 (1968).

[10] T. Shutt et. al. Phys. Rev. Lett. 693425 (1992).

[11] A.R. Sattler et al. Phys. Rev. 143588 (1966).

[12] L. Baudis et al. Nucl. Instrum. Methods Phys. Res. A 418 (1998) 348-354. 
[13] E. Simon et. al. Nucl. Instrum. Methods Phys. Res. A 507 (2003) 643-656.

[14] Kenneth S. Krane, Introductory Nuclear Physics, 1987, p. 341 published by John Wiley \& Sons, Inc.

[15] A. Dragic et. al., 2013 J. Phys.: Conf. Ser. 409012054 doi:10.1088/1742$6596 / 409 / 1 / 012054$.

[16] GEANT4, S. Agostinelli et al., Nucl. Instrum. Meth. A506, 250 (2003).

[17] Princetion Gamma Tech Inc.

[18] Sources, QSA Global Inc.

[19] SAINT-GOBAIN Crystals.

[20] R. Wordel et. al., NIM A369(1996)557.

[21] G. Fehrehbacher, R. Meckbach and H. G. Paretzke, NIM A372(1996)239.

[22] G. Fehrehbacher, R. Meckbach and H. G. Paretzke, NIM A372(1996)391.

[23] E. Gete et. al., NIM A388(1997)212.

[24] K. W. Geiger and L. Van Der Zwan, Nuclear Instruments and Methods 131 (1975) 315-321.

[25] F. De Guarrini and R. Malaroda, Nuclear Instruments and Methods 92 (1971) $277-284$.

[26] X-Ray Instruments Associates, 8450 Central Ave., Newark CA 94560, USA.

[27] Wavemetrics Inc., PO Box 2088, Lake Oswego, OR 97035, USA.

[28] Rene Brun and Fons Rademakers, Nucl. Instr. and Meth. 389, 81 (1997); http://root.cern.ch.

[29] J.E. Moyal, Theory of ionization fluctuations, Phil. Mag. 46 (1955) 263.

[30] H. Bethe und J. Ashkin in "Experimental Nuclear Physics, ed. E. Segré, J. Wiley, New York, 1953, p. 253.

[31] M.J. Berger, J.S. Coursey, M.A. Zucker, and J. Chang (2005), ESTAR, PSTAR, and ASTAR: Computer Programs for Calculating Stopping-Power and Range Tables for Electrons, Protons, and Helium Ions (version 1.2.3). [Online] Available: http : //physics.nist.gov/Star. National Institute of Standards and Technology, Gaithersburg, MD.

[32] Refer to problem 957 in the Geant4 Problem Tracking System: http :

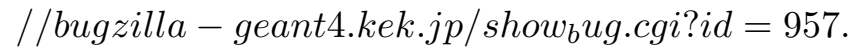

[33] A. J. Koning, S. Hilaire and M. C. Duijvestijn, AIP Conf. Proc., 769, 11541159 (2005).

[34] Leong Ying, Princeton Gamma-Tech Instruments (Private communication). 
[35] Refer to http ://ie.lbl.gov/toi/nuclide.asp?iZA=950241.

[36] J. W. Marsh, D. J. Thomas and M. Burke, Nuclear Instruments and Methods in Physics Research A 366 (1995) 340-348.

[37] D.-M. Mei, S. R. Elliott, A. Hime, V. Gehman and K. Kazkaz, Phys. Rev. C77, 054614 (2008). 